\title{
Remote Monitoring System of Water Supply Pipeline Based on ZigBee and WiFi
}

\author{
Yong Tie ${ }^{1}$, Ting Zhao ${ }^{1}$, Qingxia Feng ${ }^{1}$, Yang Liu ${ }^{1}$, Xueli Ren ${ }^{1, *}$ \\ 1. College of Electronic Information Engineering, Inner Mongolia University \\ tieyongs@163.com, zhaotingimu@163.com, qingxiafeng@163.com, yangliuimu@163.com,renxueli@imu.edu.cn
}

\begin{abstract}
Water is an extremely important resource for the man, thus, to improve the reliability of water supply pipeline is very useful. In this paper, a remote monitoring system for water supply pipeline based on ZigBee and WiFi is designed. The system can effectively resolve the problems of poor real-time performance, high cost, and labor intensiveness of conventional water supply pipeline leakage monitoring systems. The system can be used to detect leaks in the water supply pipe based on the transient change of the negative pressure in the pipe. When the water supply pipeline leaks, the sound signal in the pipeline is collected, and the acoustic signal data are transmitted to the data processing center in real time through the wireless sensor network and the WiFi network. By analyzing and processing these data in the host computer, the leak is located and the goal of monitoring the water supply pipeline in real time is eventually reached.
\end{abstract}

Keywords-Water supply pipeline; ZigBee; WiFi; Negative pressure wave method

\section{INTRODUCTION}

Water is the source of life, but water resources are not inexhaustible. Water resources are deteriorating due to the increase in population worldwide and the increase in environmental pollution. In addition, the aging, corrosion, and man-made damages of water supply pipelines can result in huge waste of water resources. Leakages of water supply pipelines are common problems in China's water supply industry $[1,2]$. According to statistics, the leakage rate of urban water supply pipelines in China is between $10 \%$ and $30 \%$, with an average of $15 \%$. In comparison, the water leakage rate in developed countries is far less than that of China owing to their advantages in economy and science and technology. Leaks in water supply pipelines will not only waste water resources, but also restrict economic development [3]. For this reason, designing a water supply pipeline monitoring system is of great significance.

The traditional water supply pipeline monitoring technology focuses on the hardware method. The detection of leaks is mainly accomplished by performing external examination or the insertion and removal of testing instrument from the pipeline. With the development of Internet of Things technology, these traditional time-consuming and laborintensive methods are gradually phased out. Nowadays, leakage monitoring methods that combine hardware and software have appeared in the field of water supply pipeline leakage monitoring [4 8]. Jawhar et al. proposed a framework for a wireless sensor network to monitorwater supply pipelines. It consists of sensor nodes that capture information inside and around the pipeline. The data collected by these sensor nodes are first sent to the gateway node and then to the base station after processing. Localization algorithms are then used to determine the location of the leaks [9 11]. Michael Allen et al. developed a system based on a wireless sensor network that uses ARM chips as the core and uses 3G communication technology to comprehensively acquire various data, including water pressure, water flow velocity, and water quality in the pipeline to achieve leak monitoring and localization, auxiliary maintenance decision-making, and water use strategy [12,13]. The software and hardware involved in the above two systems are complex and the development and deployment costs are high, which are not conducive to large-scale deployment of wireless sensor networks.

To address the leakage problem of water supply pipeline, a practical ZigBee and WiFi-based water supply pipeline monitoring system is developed in this paper. The system uses a wireless sensor network, data fusion technology, and communication and other technologies to achieve the capability of acquiring and processing water supply pipeline signals in real time. The system can not only avoid the inconveniences caused by manual monitoring, but has many advantages such as low power consumption, easy installation and configuration, low maintenance cost, low construction cost, and large capacity.

The rest of the paper is organized as follows. The architecture of the proposed system is briefly introduced in Section II. In Section III, the hardware of the system is described. The software of the system is presented in Section IV. And we give conclusion in Section V.

\section{SySTEM DESIGN}

\section{A. System Design}

The ZigBee and WiFi-based water supply pipeline remote monitoring system designed in this paper consists mainly of three parts, namely, a ZigBee wireless communication network, an ARM gateway and a center computer [14 17]. The overall framework of the system is shown in Fig. 1. In the system, the sensors are used to acquire the signal of the pipeline. Then, the received signals of the sensors are transmitted to the ARM gateway through the ZigBee wireless communication network. The ARM gateway transmits the signal to the center computer to detect the condition of the water supply pipelines.

The ZigBee wireless communication network consists of a coordinator, a router and four terminal node devices. The terminal node device equipped with the sound sensor acquires the acoustic signal in the pipeline. The router is responsible for the routing and forwarding of the data. The coordinator 


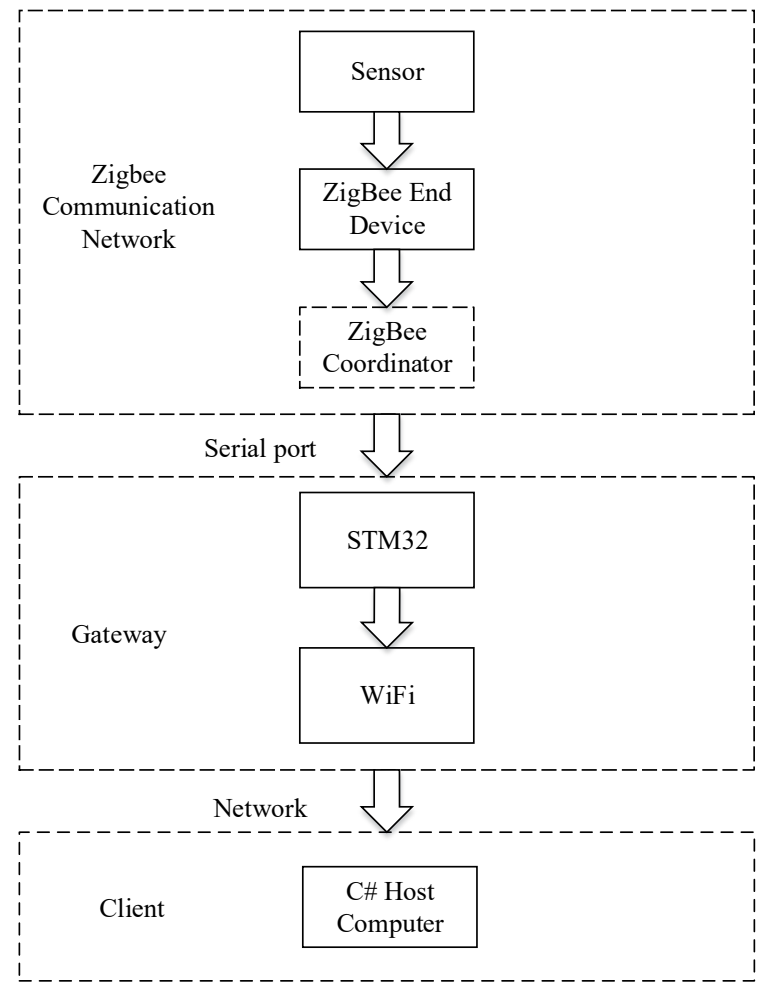

Fig. 1. Block diagram of the system structure.

establishes a connection with the ARM gateway through the serial port and transmits the acquired sensor data information to the ARM gateway to achieve a two-way transmission of data between the ZigBee wireless communication network and the ARM gateway. The ARM gateway is the communication hub of the entire water supply pipeline remote monitoring system. It acts as a connection between the ZigBee wireless communication net-work and the host computer. The host computer monitors and controls the function of the ZigBee wireless communication network via the serial communication between the ARM gateway and the ZigBee coordinator.

The application program of the central computer displays the interactive program of the entire water supply pipeline remote monitoring system. It gives the system the ability to read, acquire, store and display data.

\section{B. ZigBee And WiFi Technology}

The networking function of the water supply pipeline monitoring system designed in this paper relies on wireless network technology. The current mainstream wireless communication technologies are Bluetooth, ZigBee and WiFi technology. Compared with other wireless communication technologies, ZigBee provides secure, reliable and low-power wireless network communication [18]. This is why the ZigBee technology is employed in this paper for monitoring the water supply pipeline system. The coordinator is surrounded by a set of terminals or router devices in a star network topology, and this topology is popular because of its simplicity. However, all communication paths must pass through the coordinator, so the entire network will stop working when the coordinator stops operating. Similarly, the coordinator can easily become a bottleneck for traffic within the network. If a router fails, all nodes of the router cannot communicate with the rest of the network in a tree network topology, therefore this type of topology is not the most reliable topology. Assuming a particular router fails, ZigBee's self-healing mechanism will allow the alternate path to search for messages in a mesh network topology, so the mesh topology is the most flexible of the three topologies.

In addition, the system also needs to implement remote monitoring, whereas ZigBee technology can only transmit data over short range. For this reason, WiFi technology is also used in this paper to transmit data to the server.

ZigBee has a variety of networking modalities, and they can be divided into three types of topological structures - the star topology, the mesh topology, and the tree topology. The system here combines the characteristics of the three network structures of ZigBee, taking into account the application environment of water supply pipeline, and choosing the mesh network as the topological structure of the ZigBee communication network.

\section{SystemFunction Analysis}

Wireless Sensor Network is widely used in many fields, including medical, environmental monitoring, security, military and other fields. The ZigBee and WiFi-based water supply pipeline remote monitoring system proposed in this paper is the specific application of wireless sensor network in the field of environmental monitoring. The system includes the following functions. The first one is collecting sound signals in real-time in the water supply pipeline. The second is controlling multiple ZigBee nodes remotely in the water supply pipeline. In the third stage the host computer displays the data collected by the sensor in real-time and stores it in the database, so that computer can call the required data through the leak point location algorithm. In the end, Networking function, which is convenient for relevant personnel to view data through any networked computer. In addition, since the system is arranged in the water supply pipeline, the system is easy to arrange, takes up little space, and the cost is controllable.

\section{PRINCIPLES OF SYSTEM HARDWARE}

This paper uses a negative pressure wave method to determine whether the water supply pipeline leaks. The principle is as follows: negative pressure wave phenomena are easy to occur in the water supply pipeline. The propagation speed of the negative pressure wave can generally reach 1000$2000 \mathrm{~m} / \mathrm{s}$. Since the negative pressure wave can be captured by a dynamic pressure sensor, a pressure sensor can be installed at each end of a section of the pipeline to monitor whether a negative pressure wave is generated, and to determine whether the water supply pipe leaks.

The entire water supply pipeline monitoring system can be divided into four sections. The first part is a monitoring network consisting of water supply pipeline monitoring nodes. In this network, both the router and the terminal node are composed of water supply pipeline monitoring nodes. The gateway consists of a coordinator node and an STM32 controller $[19,20]$. The functions of the monitoring node 
include three parts: first, the sound generated in the leaky pipeline, the acquisition of the pressure signal, and the analog-

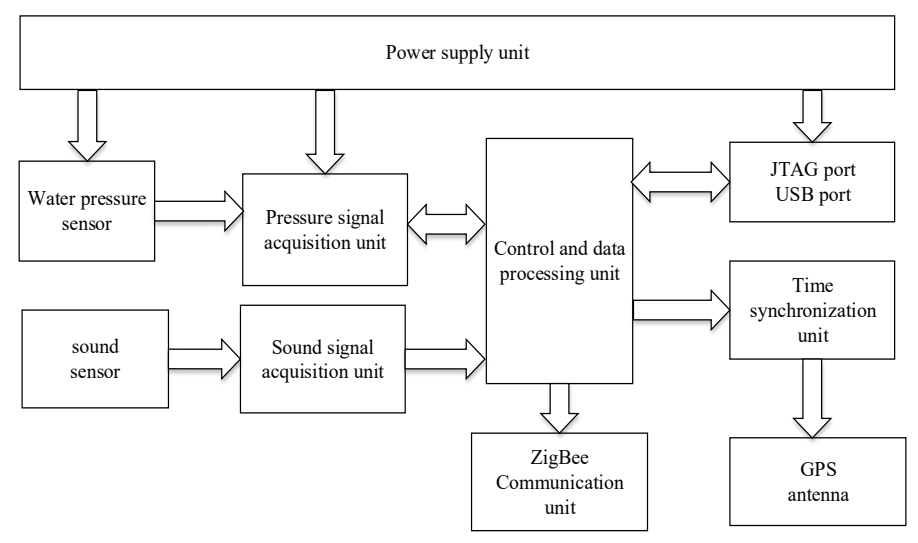

Fig. 2. Pipeline monitoring node structure.

to-digital conversion; second, the storage of the waveform data of the sound signal; and third, the communication with other nodes.

The monitoring node is mainly composed of a power supply unit, a water pressure sensor, a sound sensor, a pressure signal acquisition unit, a control and data processing unit, a time synchronization unit, a ZigBee communication unit and a GPS antenna, asontrol theory, as shown in Fig. 2.

The water supply pipeline leakage monitoring node is an important part of the entire water supply pipeline monitoring system. Since the localization method for the leak is based on the time difference between the leak and the two nodes at the ends, this requires that the two end nodes must be synchronized otherwise the determination of the leak location will be inaccurate due to time error. Therefore, each node needs to have a time synchronization module.

The second part is the gateway that consists of the coordinator node, the STM32 controller and the WiFi module. In addition to the Z-Stack protocol stack and the OSAL operating system, the ZigBee coordinator also disassembles the

physical layer frames transmitted from the end nodes, extracts the acquired information, and then transmits the information through the serial port to the STM32.

The third part is the cloud server. The system transmits the data to the cloud server platform through the WiFi module to prepare the host computer, developed in $\mathrm{CH}$, for displaying and processing the data.

The fourth part is the data processing center. It must be able to display, store and recall the acquired data, to further analyze and process the data, and to issue an alarm when an abnormal situation occurs.

\section{System Software Design}

\section{A. ZigBee Network Design}

In this system, the design of the ZigBee wireless communication network has three parts: ZigBee coordinator node software design, ZigBee router node software design and
ZigBee terminal node software design. Among them, the ZigBee coordinator is the core of the ZigBee wireless communication network. Its main functions include building and maintaining the network, controlling the ZigBee terminal node and making the ZigBee router node to join the network and to accept the data sent by the node. In addition, the ZigBee coordinator is connected to the ARM gateway via a serial port and communicates with the ZigBee terminal devices (or ZigBee router devices) through a wireless sensor network.

The ZigBee router acts as a relay in the wireless communication network. It mainly sends data to the ZigBee coordinator in cooperation with the ZigBee terminal node, thereby increases the communication distance between the nodes. The ZigBee terminal node is responsible for transmitting the data information collected by the sound sensor to the coordinator through the wireless sensor module.

\section{B. ARM Gateway Software Design}

The development software of ARM gateway in this paper is Keil. The Keil software is an integrated development environment for developing STM32 applications; it affords great advantages in source code editing. The water supply pipeline remote monitoring system developed here uses the 4.32 version of the Keil software. The WiFi module ESP8266 uploads data to the server via the TCP protocol. Once a TCP connection is established, data can be reliably transmitted between devices.

This system must not only monitor water supply pipeline in a local area network, but also monitor it in a wide area network. In order to achieve this capability, the system uses a peanut shell software to map the IP address of the intranet to the external network. After the intranet is successfully mapped to the external network, the software program of the gateway needs to be designed. Fig. 3 shows the working flow chart of the ARM gateway software.

The ARM gateway is the communication hub of the entire water supply pipeline remote monitoring system. It plays an important role in the connection between the ZigBee communication network and the host computer. At first, initializing each port of STM32. And then, Configuring the WiFi module to establish a TCP connection with the host computer. After the serial port 1 is interrupted, the gateway executes the control command which is sent by the host computer, and sends the command to the ZigBee coordinator through the serial port 2. After the serial port 2 is interrupted, the STM32 port receives the data sent by the coordinator. At last, STM32 sends the data to the host computer through the WiFi module.

\section{Host Computer Software Design}

The development environment of the host computer for this system is Visual Studio 2012, which allows developers to develop programs in $\mathrm{CH}, \mathrm{C}++, \mathrm{VB}, \mathrm{html}$, javascript and other languages. The host computer interface of the water supply pipeline monitoring system is designed mainly with Windows application and developed in $\mathrm{C \#}$ language. The host computer application consists mainly of two parts: the login interface and the main interface. The main interface includes network configuration, data display, and others. The host computer 
establishes a network connection with the TCP server on the ARM gateway through the TCP protocol in order to read the sensor data information, and thereby achieve the functions of

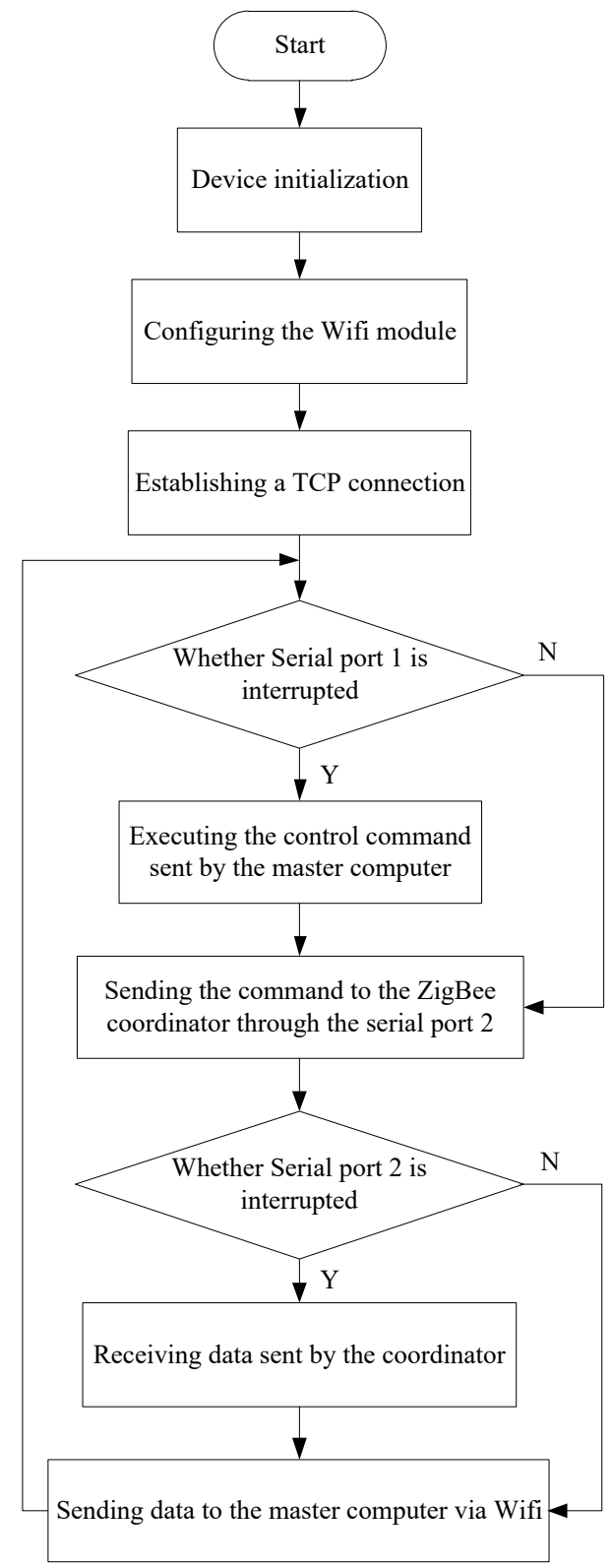

Fig. 3. Working flow chart of gateway.

data collection, storage and display. Fig. 4 shows the work flow chart of the host computer. There are five stages for the system. At the first step, Logging in to the main interface of the water supply pipeline remote monitoring system. The second step is configuring the network, starting the TCP server, and establishing a TCP connection with the ARM gateway. The host computer sends a control command to the ZigBee wireless communication network, and selects the ZigBee node of the water supply pipeline to be monitored to start working. Receiving data transmitted by the ARM gateway and parsing the data. Displaying the data waveform and saving the data to the database to provide data for subsequent calls to the matlab algorithm to locate leaks.

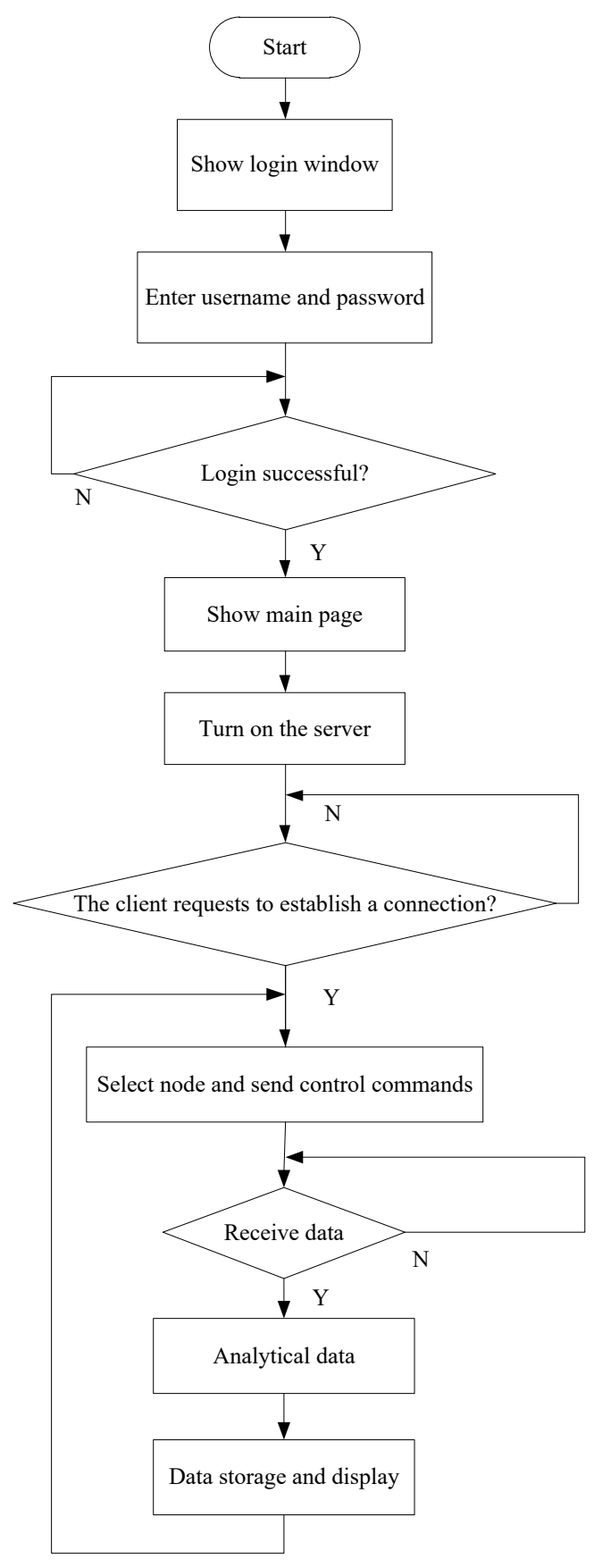

Fig. 4. Working flow chart of host computer.

\section{CONCLUSION}

In this paper, a ZigBee and $\mathrm{WiFi}$-based remote monitoring system for water supply pipeline is designed. The system utilizes the ZigBee wireless sensor network for data transmission, which effectively enhances the success rate and accuracy of data transmission. The system also uses a negative pressure wave method to detect and locate the leaks of the pipeline with a faster response and higher positioning accuracy. The test results show that the system has high precision and reliability, can effectively resolve the inconveniences of 
manual monitoring, and offers many advantages such as low power consumption and easy maintenance.

\section{ACKNOWLEDGMENT}

The authors are grateful to the Science Foundation of China for its support of this research. This work was supported by the National Natural Science Foundation of China under Grant No.61461036 and No.61761033.

\section{REFERENCES}

[1] I. Jawhar, N. Mohamed, and K. Shuaib, "A framework for pipeline infrastructure monitoring using wireless sensor networks," Wireless telecommunications symposium, 2007, WTS 2007, IEEE, 2007, pp. 1-7.

[2] M. Allen, A. Preis, M. lqbal, et al., "Water distribution system monitoring and decision support using a wireless sensor network," Software Engineering, Artificial Intelligence, Networking and Parallel/Distributed Computing (SNPD), 2013 14th ACIS International Conference on, IEEE, 2013, pp. 641-646.

[3] J. A. Stankovic, "Research directions for the internet of things," IEEE Internet of Things Journal, vol. 1, issue 1, pp. 3-9, 2014.

[4] J. N. Al-Karaki, A. E. Jamal, and A. E. Kamal, "Routing techniques in wireless sensor networks: a survey," IEEE wireless communications, vol. 11 , issue 6, pp. 6-28, 2004

[5] M. Marks, "A survey of multi-objective deployment in wireless sensor networks," Journal of Telecommunications and Information technology, pp. 36-41, 2010

[6] C. Buratti, A. Conti, D. Dardari, et al., "An overview on wireless sensor networks technology and evolution," Sensors, vol. 9, issue 9, pp. 68696896,2009

[7] S. Siddheswar, S. Biplab, and D. Uma, Design of wireless sensor node to measure vibration and environment parameter for structural health monitoring application, Intelligent Computing and Applications, Springer, New Delhi, 2015.

[8] M. J. Chae, H. S. Too, J. Y. Kim, et al., "Development of a wireless sensor network system for suspension bridge health monitoring," Automation in Construction, vol. 21, pp. 237-252, 2012

[9] N. Sghaier, A. Mellouk, B. Augustin, et al., "Wireless sensor networks for medical care services," Wireless Communications and Mobile
Computing Conference (IWCMC), 2011 7th International, IEEE, 2011, pp. 571-576.

[10] J. Grover, "Wireless Sensor Network in Railway Signalling System," 2015 Fifth International Conference on Communication Systems and Network Technologies, IEEE, 2015, pp. 308-313.

[11] T. Alhmiedat, "An adaptive energy-efficient data collection system for ZigBee wireless sensor networks," International Journal of Distributed Sensor Networks, vol. 11, issue 12, pp. 734937, 2015.

[12] P. Spachos,L. Song, and D. Hatzinakos, "Prototypes of opportunistic wireless sensor networks supporting indoor air quality monitoring," Consumer Communications and Networking Conference (CCNC), 2013 IEEE, IEEE, 2013, pp. 851-852

[13] V. Jelicic, M. Magon, D. Brunelli, et al., "Context-adaptive multimodal wireless sensor network for energy-efficient gas monitoring," IEEE Sensors Journal, vol. 13, issue 1, pp. 328-338, 2013.

[14] H. Zhang, and W. Kang, "Design of the data acquisition system based on STM32," Procedia Computer Science, vol. 17, pp. 222-228, 2013.

[15] G. Betta, C. Liguori, A. Paolillo, et al., "A DSP-based FFT-analyzer for the fault diagnosis of rotating machine based on vibration analysis," IEEE Transactions on Instrumentation and Measurement, vol. 51, issue 6, pp. 1316-1322, 2002

[16] B. Greyvenstein, and J. E. Van Zyl, "An experimental investigation into the pressure-leakage relationship of some failed water pipes," Journal of Water Supply: Research and Technology-AQUA, vol. 56, issue 2, pp. 117-124, 2007.

[17] X. J. Wei, "Intelligent monitoring system based on STM32," e-Health Networking, Applications and Services (Healthcom), 2017 IEEE 19th International Conference on, IEEE, 2017, pp. 12-17.

[18] T. A. Alhmiedat, F. Omar, A. A. Taleb, et al., "Road safety and energy saving proposed system: A ZigBee WSN approach," International Journal of Online Engineering (iJOE), vol. 11, issue 2, pp. 55-59, 2015

[19] Z. Yong, and D. Haobin, "Design of a seismic data acquisition system based on STM32 and LabVIEW," Application of Electronic Technique, vol. 10, pp. 0-28, 2012.

[20] Y. Gao, M. J. Brennan, P. F. Joseph, et al., "On the selection of acoustic/vibration sensors for leak detection in plastic water pipes," Journal of Sound and Vibration, vol. 283, issue 3-5, pp. 927-941, 2005. 